\title{
Green Tea Induced Cellular Proliferation and the Expression of Transforming Growth Factor- $\beta 1$ in the Jejunal Mucosa of Fasting Rats
}

\author{
Thazhumpal C. Mathew ${ }^{\mathrm{a}}$ Suad M. Abdeen ${ }^{\mathrm{b}}$ Hussain Dashtic Sami Asfar ${ }^{\mathrm{c}}$ \\ ${ }^{a}$ Department of Medical Laboratory Sciences, Faculty of Allied Health Sciences, and Departments of \\ ${ }^{b}$ Pathology and ' Surgery, Faculty of Medicine, Health Sciences Center, Kuwait University, Sulaibikhat, Kuwait
}

\section{Keywords}

Fasting $\cdot$ Jejunal mucosa $\cdot$ Cell proliferation $\cdot$ Growth factors

\begin{abstract}
Objective: The aim of this study was to understand whether or not the protective effect of green tea after fasting-induced damage in the jejunal mucosa of rat is dependent on cell proliferation and the stimulation of specific growth factors. Materials and Methods: Sixty adult male Wistar rats were used in this study. The animals were divided randomly into 5 groups, with 12 in each group (G1-5). The animals in G1 (control group) were fed a rat chow diet and water ad libitum. The animals in $\mathrm{G} 2$ (fasting group) were fasted for 3 days. The animals in the G3, G4, and G5 groups were fasted for 3 days as $G 2$, but were given water (G3), green tea (G4), or a vitamin $E$ (G5) solution, respectively, for another 7 days. The animals were euthanized, and the jejunum was removed and processed for histological and immunohistochemical analysis. Results: Compared to the G3 group, the jejunal mucosa of G4 rats showed a 70.6\% higher level $(p<0.001)$ of expression of proliferating cell nuclear antigen and $98 \%$ higher level ( $p=0.0001$ ) of the expression of transforming growth factor- $\beta 1$ (TGF- $\beta 1$ ), whereas the level of fibroblast growth factor-1 (FGF-1) and insulin-like growth factor-1
\end{abstract}

\begin{tabular}{ll}
\hline KARGER & (c) 2017 S. Karger AG, Basel \\
$\begin{array}{l}\text { E-Mail karger@karger.com } \\
\text { www.karger.com/mpp }\end{array}$ & $\begin{array}{l}\text { This is an Open Access article licensed under the terms of the } \\
\text { Creative Commons Attribution-NonCommercial 3.0 Un- } \\
\text { ported license (CC BY-NC) (www.karger.com/OA-license), } \\
\text { applicable to the online version of the article only. Distribu- } \\
\text { tion permitted for non-commercial purposes only. }\end{array}$
\end{tabular}

(IGF-1) expression was 22 and $11 \%$ lower, respectively, in G4 animals as compared to $\mathrm{G} 3$ rats. These differences in the expression of FGF-1 and IGF-1 in G4 animals were not statistically significant. Conclusion: In this study, green tea repaired the fasting-induced damage in the jejunal mucosa of rats, mainly by inducing a significant expression of TGF- $\beta 1$ in the jejunal mucosa.

(c) 2017 S. Karger AG, Basel

\section{Introduction}

Epithelial cells of the intestinal mucosa are sensitive to various pathological and physiological conditions [1-3]. In experimental animals that were fasted for 3 days, there was a decrease in the overall mucosal weight and decrease in the number and length of intestinal microvilli, resulting in the erosion and atrophy of the mucosal epithelium $[1,4]$. However, the mucosal epithelium of rats given green tea or vitamin $\mathrm{E}$ for 7 days following fasting showed a recovery to nearly normal histology from the fastinginduced damage [1]. In addition, these conditions can also lead to the erosion and atrophy of the intestinal mucosa [1-3], surgical stress [1], ischemia/reperfusion [3], trauma, burn [5], hemorrhagic shock, acute pancreatitis,

Prof. Thazhumpal C. Mathew, MSc, PhD, FRCPath Department of Medical Laboratory Sciences Faculty of Allied Health Sciences, Kuwait University PO Box 31470, Sulaibikhat 90805 (Kuwait) E-Mail tcmkwt@gmail.com 
ischemic bowel disease, mesenteric vascular occlusion, solid organ transplantation, especially small bowel transplantation [6], zinc deficiency [7], and deprivation of glutamine [8], treatment with anticancer drugs and antibiotics [9], and aging [10]. It has been shown that disruption of the integrity of the intestinal mucosa leads to mucosal and vascular permeability and bacterial translocation that ultimately results in systemic inflammation, respiratory failure, and multiple-organ failure [11].

Various studies have shown the involvement of free radicals [2] and inflammatory cytokines $[2,11,12]$ in intestinal injury and dysfunction. In support of this, we demonstrated that green tea, a potent antioxidant, protected the intestinal mucosa from fasting-induced damage [2]. Vitamin $\mathrm{E}$ also produced morphological and biochemical changes similar to that of green tea, but the effects were smaller [2]. Hence, it is probable that in addition to the antioxidant properties, other mechanisms could govern the beneficial effects of green tea in the intestinal mucosa.

Several investigators have shown the involvement of growth factors in the modulation of intestinal inflammation and repair $[11,12]$. It is possible that, in addition to its antioxidant effects, green tea could directly or indirectly induce specific growth factors that influence cellular proliferation, differentiation, and angiogenesis in the intestinal mucosa. Although several growth factors that are relevant in protecting and repairing the intestinal mucosa have been identified $[11,12]$, in this project we studied the expression of selected growth factors, such as fibroblast growth factor-1 (FGF-1), insulin-like growth factor-1 (IGF-1), and transforming growth factor- $\beta 1$ (TGF- $\beta 1$ ), which have established functions in the intestinal mucosa [13-17]. Exogenous epidermal growth factor (EGF) promotes intestinal growth. However, endogenous production of EGF by the normal colon and small intestine is minimal and the expression of EGF does not appear to be altered in patients with intestinal disorders $[18,19]$. Hence, in the current study the expression of EGF expression in the jejunal mucosa was not analyzed. Thus, the objective of this study was to examine the role of green tea in cellular proliferation and on the expression of FGF-1, IGF-1, and TGF- $\beta 1$ in the jejunal mucosa of fasting rats in comparison with that of vitamin $\mathrm{E}$.

\section{Materials and Methods}

Animals and Experimental Procedures

This study used 60 male Wistar rats weighing approximately 250-300 g that were raised in the Animal House of the Faculty of Medicine, Kuwait University, Kuwait. The facility is air condi- tioned and the temperature is maintained between 21 and $24^{\circ} \mathrm{C}$, with controlled illumination providing a 12 -h light/dark cycle. The rats were fed a standard laboratory diet (France Nutris Co. S.A., Paris, France) with water ad libitum. Each animal was housed in a separate cage. The animals were divided into 5 groups (G1-5), containing 12 animals in each group. All animal experiments were carried out in accordance with the National Institutes of Health guide for the care and use of laboratory animals. The experiments performed in the different groups of animals are given below.

G1 (control group) animals had free access to rat chow and water. The animals in G2 (fasting group) were fasted for 3 days and were used to study fasting-induced damage to the intestinal mucosa; they received only $40 \mathrm{~mL}$ of $10 \%$ glucose each day by intraperitoneal injection. G2 animals were killed after 3 days of fasting. The groups G3, G4, and G5 were also fasted as described for G2, but were given water (G3), green tea (G4), or vitamin E (G5) solution, respectively, for another 7 days. Also, the intraperitoneal administration of $10 \%$ glucose, $40 \mathrm{~mL}$ daily, was continued during this period in the G3, G4, and G5 animals. Groups G4 and G5 were used to study the effects of green tea and vitamin E, respectively, as compared with G3 animals.

The control and experimental animals were euthanized with an excess of ether and 2 inches of the jejunum was removed. The lumen of the jejunum was immediately cleaned by injecting phosphate-buffered saline through one end of the bowel using a $20-\mathrm{mL}$ syringe. Similarly, initial fixation of the intestine was carried out by injecting $10 \%$ of formalin through the bowel. The specimens were further fixed overnight in the same fixative.

\section{Preparation of Green Tea and Vitamin E Solutions}

Green tea was prepared as previously described [1-3]. Briefly, the preparation of the tea was as follows. Six grams of locally purchased dry green tea leaves were added to $1 \mathrm{~L}$ of boiling water. The preparation was allowed to simmer for a few minutes then left to cool to room temperature. The green tea solution was then poured into the animal's feeding bottle that replaced the animal's drinking water during the period of the experiment. Every morning the rats were supplied with freshly prepared green tea.

Vitamin E was prepared using capsules containing $400 \mathrm{IU}$ of d1-a-tocopheryl acetate (Vita Health Company Ltd, Winnipeg, $\mathrm{MB}$, Canada). The contents of 2 capsules (800 IU) were poured into the feeding bottles containing $500 \mathrm{~mL}$ of drinking water. The concentration of vitamin $\mathrm{E}$ was $1.6 \mathrm{IU}$ per $\mathrm{mL}$ of the solution. On average each rat consumed about $40 \mathrm{~mL}$ of green tea or vitamin $\mathrm{E}$ solution per day.

\section{Histopathological and Immunohistochemical Analysis}

The fixed specimens of jejunum from each experimental group were cut longitudinally and 2 -mm cross sections were processed and embedded in paraffin blocks. The blocks were cut into 4 - $\mu$ m-thick sections. The sections were stained for histopathologic and immunohistochemical studies. The antibody used against proliferating cell nuclear antigen (PCNA) was a mouse monoclonal antibody, while the antibodies used against FGF-1, IGF- 1 , and TGF- $\beta 1$ were affinity purified polyclonal antibodies. TGF- $\beta 1$ antibody detects the precursor and mature TGF- $\beta$, and does not cross-react with the latency-associated peptide. Antibodies against PCNA, FGF-1, and TGF- $\beta 1$ were obtained from Santa Cruz Biotechnology Inc. (Santa Cruz, CA, USA). Antibody against IGF-1 was obtained from Lab Vision Corporation (Fre- 
Fig. 1. Immunohistochemical expression of PCNA, the marker of cellular proliferation in the jejunal mucosa (a; counterstained with Mayer's hematoxylin, $\times 100$ ) and a semiquantitative analysis of the results (b). G1, control; G2, 3 days fasting; G3, 3 days fasting then 7 days water; G4, 3 days fasting then 7 days green tea; G5, 3 days fasting then 7 days vitamin E. * G4 and $\mathrm{G} 3, p<0.001 ; \mathrm{G} 5$ and $\mathrm{G} 3, p<0.001 ; \mathrm{G} 1$ and G3, $p<0.001 ; \mathrm{G} 4$ and G5, $p<0.05 ; \mathrm{G} 1$ and $\mathrm{G} 2, p=$ not significant.

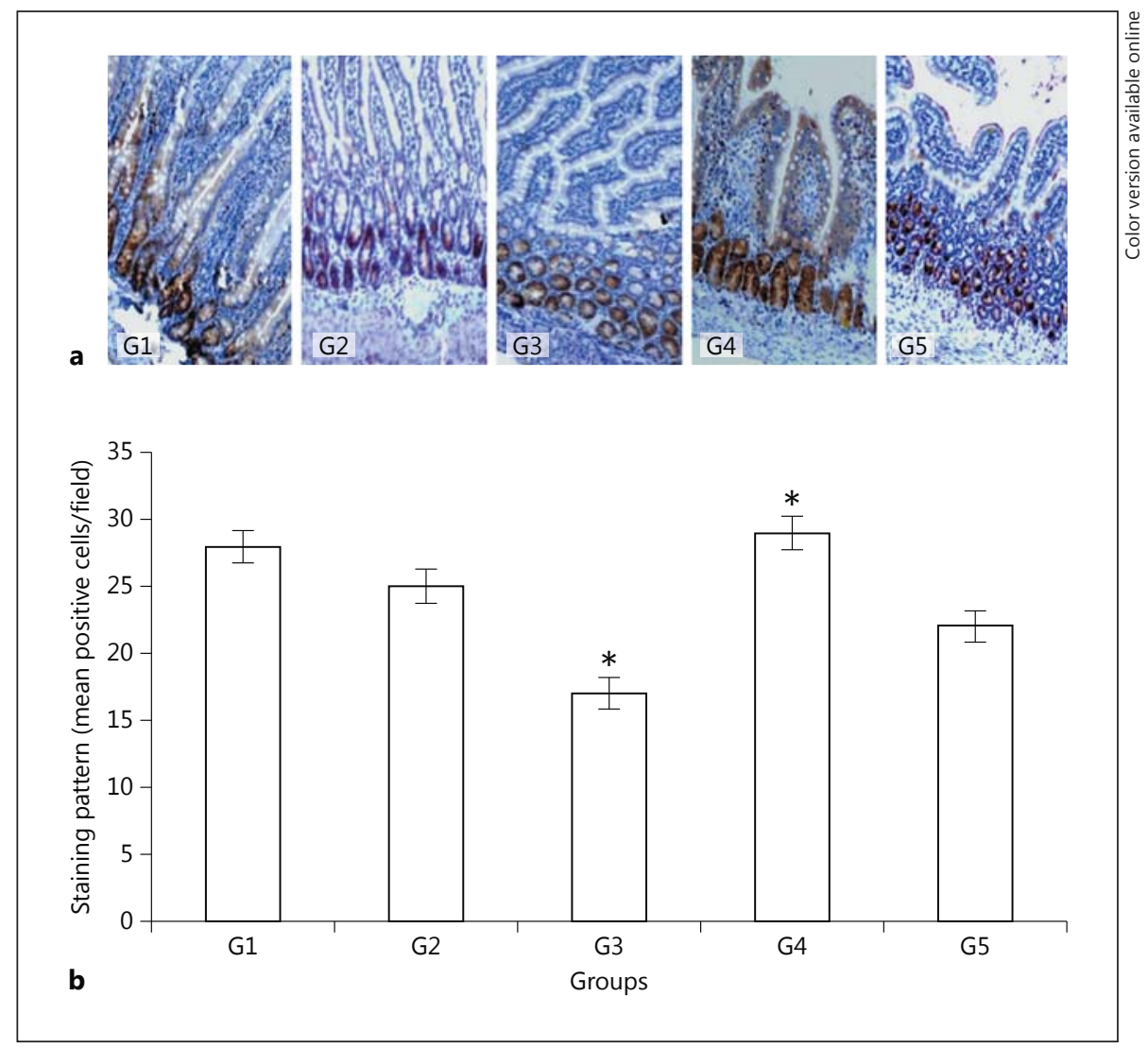

mont, CA, USA). Immunohistochemical analysis of PCNA, FGF1 , IGF- 1 , and TGF- $\beta 1$ were carried out using the $\mathrm{LSAB}^{\mathrm{TM}}+\mathrm{Kit} /$ HRP (Dako North America Inc., Carpinteria, CA, USA), which utilizes a refined avidin-biotin technique in which a biotinylated secondary antibody reacts with several peroxidase-conjugated streptavidin molecules.

For immunohistochemical techniques, the sections were transferred to $0.05 \mathrm{~mol} / \mathrm{L}$ Tris-buffered saline (TBS) at $\mathrm{pH}$ 7.6, dewaxed with xylene, and hydrated using a descending series of alcohols. The endogenous peroxidase activity in the tissue was removed by incubating the slides in hydrogen peroxidase for $10 \mathrm{~min}$. Subsequently, the sections were washed with water for $5 \mathrm{~min}$. The sections were then subjected to the antigen retrieval step (microwave treatment with citrate buffer at $\mathrm{pH} \mathrm{6)}$ as per the manufacturer's instructions (Dako North America Inc., Carpinteria, CA, USA) [20]. To block the nonspecific binding of the antibody, the slides were rinsed in TBS for $5 \mathrm{~min}$ and then covered with normal serum for $10 \mathrm{~min}$. The sections were then incubated with the appropriate primary antibodies at specific dilutions as per the manufacturer's instructions [20]. The sections were then incubated with biotinylated link antibody for $30 \mathrm{~min}$. After incubation with the link antibody, the sections were treated with streptavidin-HRP for $30 \mathrm{~min}$. All these incubation steps were performed with interposed washing steps with TBS between the changes from one reagent to the other. Finally, the sections were treated with diaminobenzene for a few minutes. The peroxidase of the streptavidin-HRP reacts with

Green Tea and the Induction of Growth Factors diaminobenzene to produce, via oxidation and polymerization, an insoluble brown product at the site of the target antigen. The sections were counterstained with Mayer's hematoxylin and washed under running tap water for about 5 min until the nucleus became blue. The sections were dehydrated, cleared, and mounted in a synthetic resin mounting media containing a mixture of distyrene, a plasticizer, and xylene (DPX). The primary antiserum was omitted for negative controls and appropriate tissues were used as positive controls.

All the sections were examined using a Zeiss light microscope (Carl Zeiss AG, Oberkochen, Germany) by a members of the team (T.C.M) who was blinded to the different animal groupings. They were again double-checked by another member of the team (S.M.A) who was also blinded to the different animal groupings. For semiquantification of the immunohistochemistry results, 30 fields per section were examined. All immunopositive cells of relatively similar intensity were counted in each field. The mean \pm standard error of positive cells in 30 fields was calculated for each group.

\section{Data Analysis}

Data were analyzed using ANOVA-factorial analysis of the StatView 4.5 computer package (Abacus Concepts, Berkeley, CA, USA) and $p<0.05$ was considered to show a significant difference between groups. The results are expressed as the mean value \pm SEM. 
Table 1. Percentage difference of the mean of growth factors based the level of expression in G1 and G3

\begin{tabular}{|c|c|c|c|c|c|c|c|}
\hline \multirow[t]{2}{*}{ Growth factor } & \multirow[t]{2}{*}{ Group } & \multirow[t]{2}{*}{ Mean } & \multirow{2}{*}{$\begin{array}{l}\text { Standard } \\
\text { error }\end{array}$} & \multicolumn{2}{|c|}{ Based on G1 } & \multicolumn{2}{|c|}{ Based on G3 } \\
\hline & & & & $\begin{array}{l}\text { difference } \\
\text { in mean }\end{array}$ & $\%$ difference & $\begin{array}{l}\text { difference } \\
\text { in mean }\end{array}$ & $\%$ difference \\
\hline \multirow[t]{5}{*}{ FGF } & G1 & 20.625 & 0.778 & - & - & 18.375 & 816.667 \\
\hline & $\mathrm{G} 2$ & 13.500 & 1.569 & -7.125 & -34.545 & 11.250 & 500.000 \\
\hline & G3 & 2.250 & 0.366 & -18.375 & -89.090 & - & - \\
\hline & G4 & 1.750 & 0.250 & -18.875 & -91.515 & -0.500 & -22.222 \\
\hline & G5 & 1.125 & 0.295 & -19.500 & -94.545 & -1.125 & -50.000 \\
\hline \multirow[t]{5}{*}{ IGF } & G1 & 39.750 & 1.250 & - & - & 3.500 & 9.655 \\
\hline & G2 & 20.917 & 1.443 & -18.833 & -47.378 & -15.333 & -42.298 \\
\hline & G3 & 36.250 & 1.411 & -3.500 & -8.805 & - & - \\
\hline & G4 & 32.250 & 0.996 & -7.500 & -18.867 & -4.000 & -11.034 \\
\hline & G5 & 22.000 & 0.567 & -17.750 & -44.654 & -14.250 & -39.310 \\
\hline \multirow[t]{5}{*}{ TGF- $\beta 1$} & G1 & 23.333 & 0.644 & - & - & 4.333 & 22.805 \\
\hline & $\mathrm{G} 2$ & 15.000 & 1.292 & -8.333 & -35.713 & -4.000 & -21.053 \\
\hline & G3 & 19.000 & 0.866 & -4.333 & -18.570 & - & - \\
\hline & G4 & 37.625 & 1.322 & 14.292 & 61.252 & 18.625 & 98.026 \\
\hline & G5 & 25.625 & 0.680 & 2.292 & 9.823 & 6.625 & 34.868 \\
\hline
\end{tabular}

G1, control; G2, 3 days fasting; G3, 3 days fasting then 7 days water; G4, 3 days fasting then 7 days green tea; G5, 3 days fasting then 7 days vitamin $\mathrm{E}$.

\section{Results}

\section{Histological Studies}

The hematoxylin and eosin figures showing the histology of the jejunal mucosa of normal and experimental groups are not shown here as they were described in our previous studies. Briefly, the histopathological changes were as follows. Control animals (G1) showed a normal mucosal pattern. In the G2 group (fasting for 3 days) there was a shortening, atrophy, fragmentation, and loss of the villi. In the G3 group (rats that were given only water for 7 days following 3 days of fasting), the villous architecture became worse with continued atrophy, fragmentation, and loss of the villi. However, the jejunal villi of G4 and G5 rats (given green tea and vitamin E, respectively, for 7 days following fasting) showed a recovery to nearly normal histology from the fasting-induced damage.

\section{Expression of PCNA in the Jejunal Mucosa}

The extent of cell proliferation in the intestinal mucosa of normal control (G1), fasting control (G2), and the other G3, G4, and G5 groups using antibodies against PCNA are shown in Figure 1a, b. Cell proliferation was $10.7 \%$ less in the jejunal mucosa of G2 rats that were fast- ed for 3 days as compared to G1 rats, whereas in the G3 rats the level of PCNA expression was 39.3\% less than that of G1. Hence, the level of PCNA expression in G3 animals was further lower than in G2 rats. However, animals that were fed with green tea showed a $3.6 \%$ higher level of PCNA than the level of PCNA expressed in normal control rats. Thus, the cell proliferation in the green tea-treated group (G4) was even higher (3.6\%) than the level of PCNA expressed in the normal control group (G1; $p<0.001)$. A similar pattern of PCNA expression was not observed in the G5 animals that were fed with vitamin E. Rats that were given green tea (G4) or vitamin E (G5) showed 70.6\% $(p<0.001)$ and $29.4 \%(p<0.001)$ more cell proliferation, respectively, as compared to the G3 group. However, the proliferation of cells in the jejunal mucosa of rats that were treated with green tea (G4) was higher than the vitamin E-treated group (G5; $p<$ 0.05).

\section{Expression of FGF-1 in the Jejunal Mucosa}

The expression of FGF-1 in the jejunal mucosa of G2 animals ( 3 days fasting) was $34.5 \%$ less $(p<0.05)$ as compared to the normal controls (G1; Table 1; Fig. 2a, b). G3, G4, and G5 animals showed 89.1, 91.5, and 94.5\% less FGF-1 expression, respectively, as compared to G1 ani- 
Fig. 2. Immunohistochemical expression of FGF-1 in the jejunal mucosa (a; counterstained with Mayer's hematoxylin, $\times 100$ ) and a semiquantitative analysis of the results (b). G1, control; G2, 3 days fasting; G3, 3 days fasting then 7 days water; G4, 3 days fasting then 7 days green tea; G5, 3 days fasting then 7 days vitamin E. G4 and $\mathrm{G} 3, p=$ not significant; G1 and G2, $p<$ 0.05; G3 and G2, $p<0.001$; G5 and G3, $p=$ not significant; G4 and G5, $p=$ not significant; G1 and G4, $p<0.0001 ; \mathrm{G} 1$ and G5, $p<0.0001$.

Fig. 3. Immunohistochemical localization of IGF-1 in the jejunal mucosa (a; counterstained with Mayer's hematoxylin, $\times 100$ ) and a semiquantitative analysis of the results (b). G1, control; G2, 3 days fasting; G3, 3 days fasting then 7 days water; G4, 3 days fasting then 7 days green tea; G5, 3 days fasting then 7 days vitamin E. G4 and G3, $p=$ not significant; G1 and G2, $p<$ 0.0003; $G 1$ and $G 3, p=$ not significant; $G 4$ and $\mathrm{G} 5, p=0.0001$.

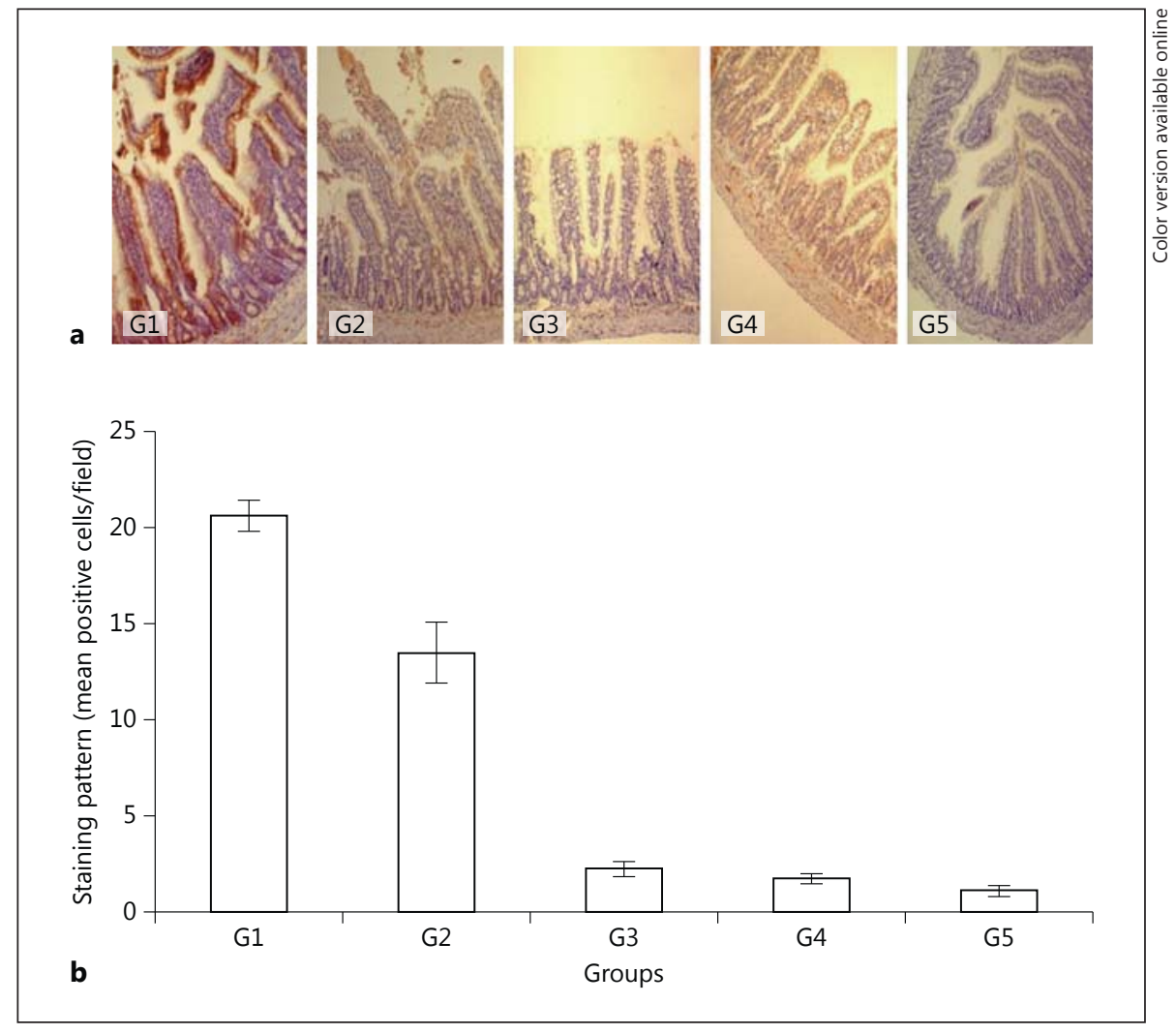

a
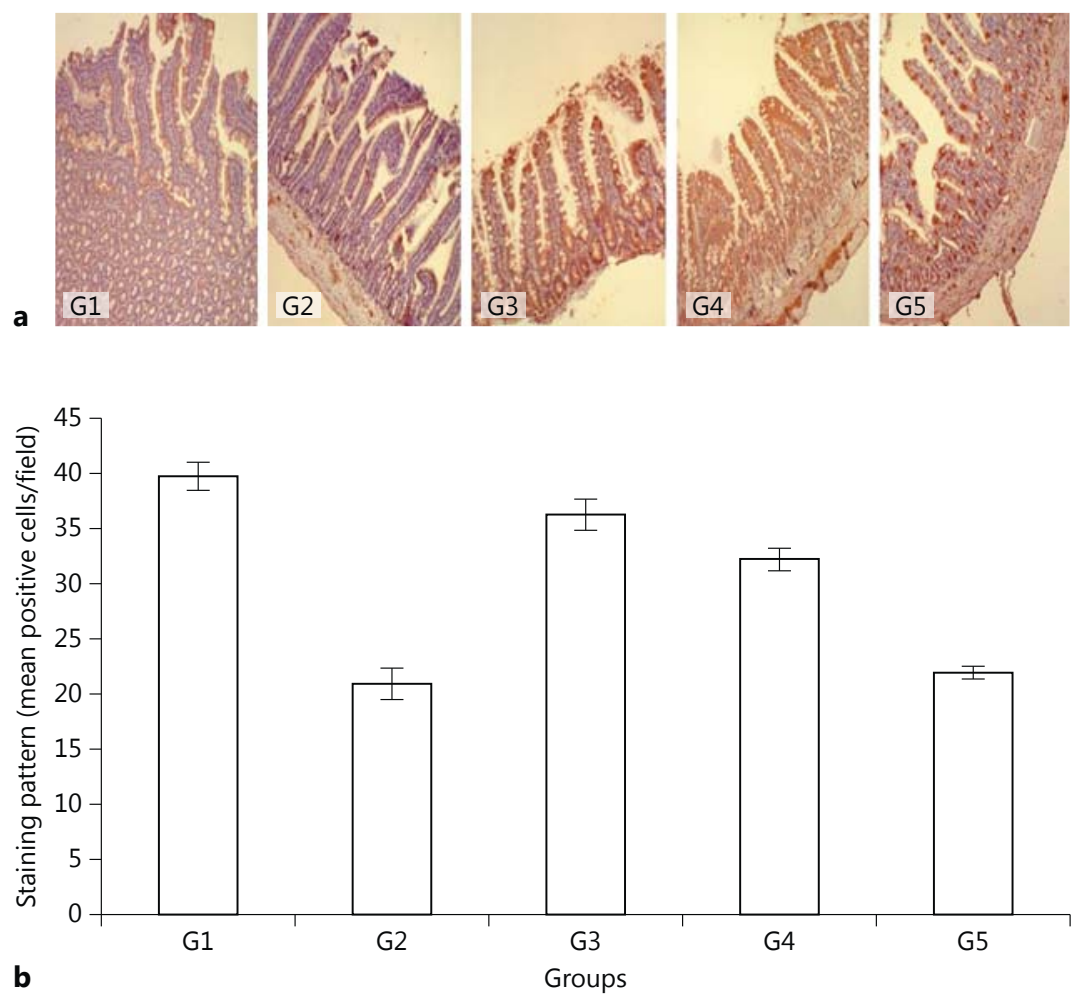

Green Tea and the Induction of Growth Factors
Med Princ Pract 2017;26:343-350 DOI: $10.1159 / 000468937$ 
Fig. 4. Immunohistochemical expression of TGF- $\beta 1$ in the jejunal mucosa (a; counterstained with Mayer's hematoxylin, $\times 100)$ and a semiquantitative analysis of the results (b). G1, control; G2, 3 days fasting; G3, 3 days fasting then 7 days water; G4, 3 days fasting then 7 days green tea; G5, 3 days fasting then 7 days vitamin E. * G4 and G3, $p<0.0001$; G1 and G2, $p<0.005$; $\mathrm{G} 1$ and $\mathrm{G} 3, p=0.003 ; \mathrm{G} 1$ and $\mathrm{G} 4, p<$ $0.0001 ; \mathrm{G} 4$ and $\mathrm{G} 5, p<0.0001$.

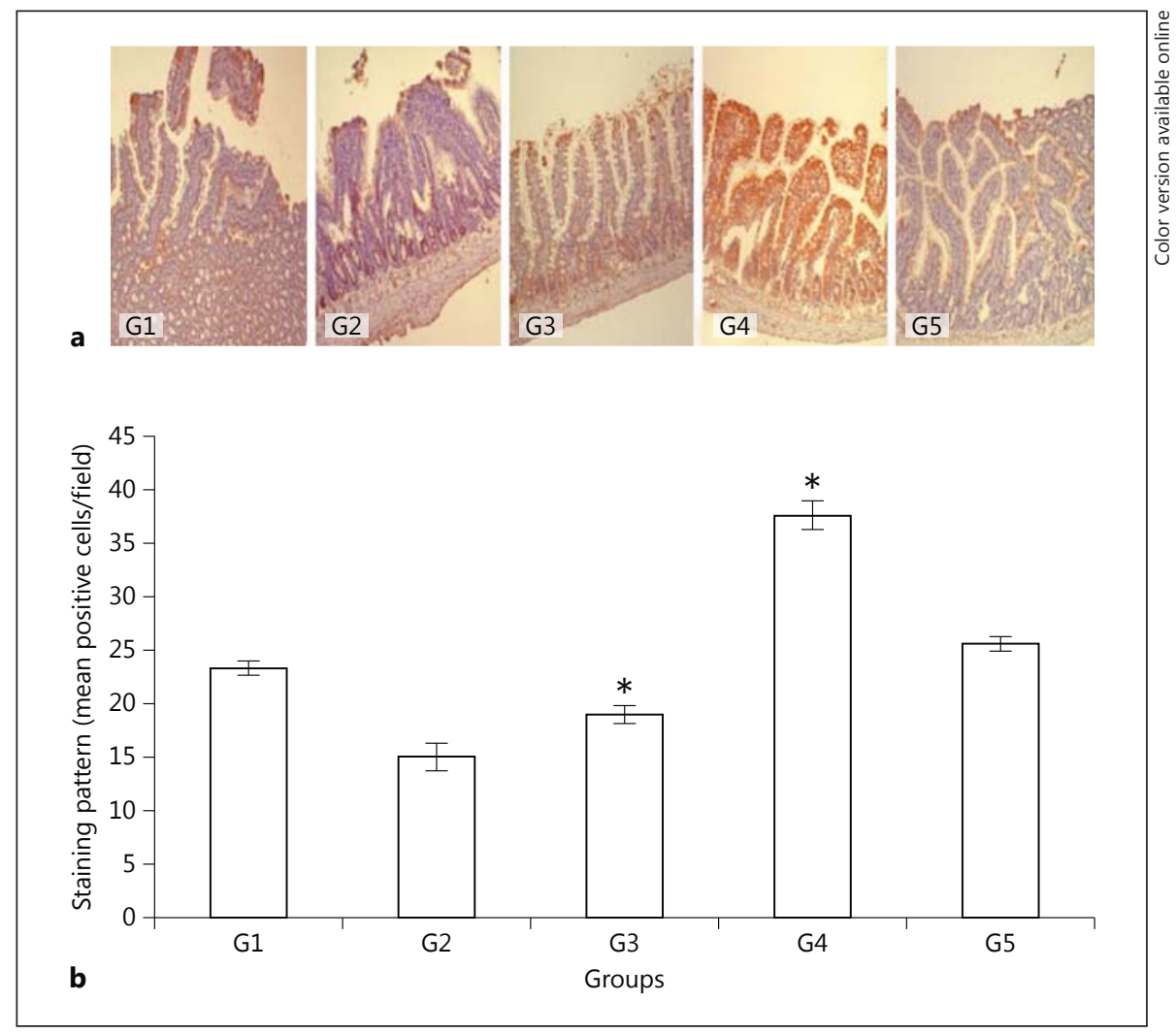

mals $(p<0.0001)$. Drinking green tea $(\mathrm{G} 4)$ or vitamin $\mathrm{E}$ (G5) for 7 days after fasting did not have any positive effect on the expression of FGF-1 in the jejunal mucosa of the rats as compared to the rats that were given only water following 3 days of fasting. These rats (G4, G5) showed 22.2 and $50.0 \%$ less FGF-1 expression, respectively, as compared to the G3 group. There was no statistically significant difference in the expression of FGF-1 between the green tea (G4) or vitamin E (G5) groups.

\section{Expression of IGF-1 in the Jejunal Mucosa}

The expression of IGF-1 was lower in G2, G3, G4, and G5 animals as compared to the G1 control group (Table 1); however, the level of IGF-1 was the lowest $(47.3 \% ; p=0.0003)$ in the jejunal mucosa of G2 rats compared to the controls (Table 1; Fig. 3a, b). There were no statistically significant differences between the expressions of IGF-1 in G3 animals, which were given only water for 7 days after 3 days of fasting, as compared to G4 animals that were given green tea. The level of IGF-1 expression in the vitamin E group (G5) was 39.3\% less, while it was only $11.03 \%$ less in green tea-fed rats $(\mathrm{G} 4 ; p=0.0001)$.
Expression of TGF- $\beta 1$ in the Jejunal Mucosa

TGF- $\beta 1$ expression in G2 and G3 rats was $35.7 \%(p<$ $0.005)$ and $18.6 \%(p<0.003)$ less, respectively, than in the controls (Table 1; Fig. 4a, b). On the other hand, the rats that were fed with green tea (G4) showed a $61.3 \%$ higher level of TGF- $\beta 1$ expression and the rats fed with vitamin E showed a 9.8\% higher level of TGF- $\beta 1$ expression than the G1 (control) rats. Drinking green tea for 7 days after 3 days of fasting (G4) resulted in a $98.0 \%$ higher level of TGF- $\beta 1$ expression as compared to the water-fed controls (G3; $p<0.0001)$. Vitamin E-treated rats (G5; Table 1; Fig. $4 \mathrm{a}, \mathrm{b})$ also showed a $34.9 \%$ higher level of TGF- $\beta 1$ expression, but to a significantly lesser extent $(p<0.0001)$ as compared to that of the green tea group (G4; Table 1; Fig. 4a, b).

\section{Discussion}

This study demonstrated that the protective and or repairing effect of green tea in the jejunal mucosa of rats after fasting-induced damage is dependent on cell proliferation and the induction of specific growth factors. 
Also, green tea induced cellular proliferation in the jejunal mucosa by stimulating TGF- $\beta 1$, which is a regulator of the cell cycle and growth [17], or similar other growth factors.

The PCNA immunohistochemical analysis showed that the level of PCNA expression was less in the jejunal mucosa of G2 and G3 animals than G1 rats, thereby indicating the low level of cell division. In addition to low cell proliferation, there was a higher level of cell death in the rat jejunal mucosa $[13,21]$. The level of PCNA expression in rats fed green tea (G4) for 7 days following 3 days fasting was $70.6 \%$, while the PCNA level in vitamin E-fed rats (G5; for 7 days following 3 days of fasting) was only $29.4 \%$ as compared to the 7-day water-fed animals (G3) following 3 days of fasting. As the effect of vitamin $\mathrm{E}$, which is a potent antioxidant, was almost $50 \%$ less than the effect of green tea, it is obvious that in addition to its antioxidant properties, green tea has other beneficial mechanisms to induce cellular proliferation to a higher level than vitamin $\mathrm{E}$.

The expression of FGF-1 in the jejunal mucosa of G2 and G3 rats was less as compared to the normal controls (G1). Apparently, drinking green tea (G4) or vitamin E (G5) for 7 days after fasting did not have any positive effect on the expression of FGF-1 in the jejunal mucosa; the expression of FGF-1 between the green tea (G4) or vita$\min \mathrm{E}$ (G5) groups was not statistically significant. Hence, both green tea and vitamin $\mathrm{E}$ did not have any role in the upregulation of the expression of FGF in the fasting animal model, although FGFs are involved in the regulation of cell proliferation, differentiation, and apoptosis [22, 23]. Similar to EGF, FGF is also implicated in tumorigenesis and metastasis [23].

The data presented in this study showed that IGF-1 expression was decreased in rats after 3 days of fasting, but the level of IGF-1 increased in the G3, G4, and G5 animals. However, the increase of IGF-1 expression in the green tea group (G4) was not significantly different from the $\mathrm{G} 3$ group. In the vitamin E group, the expression of IGF-1 was significantly lower than that of the G3 and G4 groups. Hence, IGF-1 was not specifically induced by either green tea or vitamin $\mathrm{E}$ in the fasting animal model, although IGF-1 is a potent stimulator of intestinal growth [24], and in experimental animals exogenous IGF administration was able to reverse the mucosal atrophy induced by total parenteral nutrition [25].

In this study, there was a significantly lower level of TGF- $\beta 1$ expression following 3 days of fasting, with the level of TGF- $\beta 1$ expression the highest in rats that were given green tea. Although vitamin $\mathrm{E}$ induced the expres-

Green Tea and the Induction of Growth Factors sion of TGF- $\beta 1$, it was much lower than that of the green tea-fed group. It is known that TGF- $\beta 1$ is a regulator of the cell cycle and growth [26]. It maintains tissue homeostasis by regulating cellular processes that regulate cell cycle arrest, differentiation, and apoptosis. The intestinal epithelium consists of a single layer of epithelial cells and is organized in crypt-villus functional units $[13,18]$. Progenitor cells are generated from the stem cells that are located at the bottom of the crypts. In the middle of the crypt these cells differentiate into enterocytes. These differentiated cells migrate along the crypt axis and undergo apoptosis and exfoliation at the villus tip [13]. This cytostatic regulatory effect of TGF- $\beta 1$ is highly significant in tissues such as the gut epithelium, where an imbalance in cellular homeostasis in favor of increased cell division could lead to a neoplastic growth. These processes are tightly regulated by a number of mechanisms, among which TGF- $\beta$ plays an important role by controlling the relative rates of proliferation and elimination of epithelial cells [23]. This cytostatic effect of TGF- $\beta$ is highly significant in tissues such as the gut epithelium as the disruption of the balance between cell division and cell death could lead to the development of cancer [26].

In this study, depending on the extent of cellular proliferation in the jejunal mucosa of different groups of animals, there was an increase in the mucosal epithelial expression of TGF- $\beta 1$. Thus, there was a good association between cell proliferation and the expression of TGF- $\beta 1$ in the different groups. Hence, the data on TGF- $\beta 1$ expression shown in this study confirmed the previous finding [23] that TGF- $\beta$ maintains tissue homeostasis by regulating various cellular processes that regulate cell cycle arrest, differentiation, and apoptosis. In further support of the current data, Howe et al. [16] have shown that TGF- $\beta 1$ promotes epithelial barrier integrity following exposure to proinflammatory cytokines and infectious agents [16].

\section{Conclusion}

In this study, green tea repaired the fasting-induced damage in the jejunal mucosa of rats, mainly by inducing a significant expression of TGF- $\beta 1$ in the jejunal mucosa. Therefore, targeted treatment of TGF- $\beta 1$ could lead to the development of novel therapies for several enteric diseases. However, FGF-1 and IGF-1 were not specifically induced in the rats fed with either green tea or vitamin $\mathrm{E}$ for 7 days following 3 days of fasting. Thus, FGF-1 or

Med Princ Pract 2017;26:343-350 DOI: $10.1159 / 000468937$ 
IGF-1 was not linked with green tea- or vitamin E-induced jejunal mucosal repair. This study could not demonstrate whether FGF-1 or IGF-1 were expressed earlier than 7 days. Therefore, further studies are necessary to understand whether the timing of the experiment had any effect on the expression of these growth factors, and whether a similar expression of PCNA and other specific growth factors analyzed in this study can be observed in the duodenum and ileum.

\section{Acknowledgements}

The authors would like to thank Mrs. Elizabeth Mathew, Chief Technician, Department of Medical Laboratory Sciences, Faculty of Allied Health Sciences, Kuwait University, for her expert technical advice and assistance. This project was supported by a research grant from Kuwait University (No. MS01/03).

\section{Disclosure Statement}

The authors have no conflicts of interest to declare.

\section{References}

1 Asfar S, Abdeen S, Dashti H, et al: Effect of green tea in the prevention and reversal of fasting-induced mucosal damage. Nutrition 2003;19:536-540.

2 Abdeen S, Mathew TC, Khan I, et al: Fastinginduced intestinal damage is mediated by oxidative and inflammatory responses. Br J Surg 2009;96:552-559.

3 Abdeen S, Mathew TC, Dashti HM, et al: Protective effects of green tea on intestinal ischemia-reperfusion injury. Nutrition 2011;27: 598-603.

4 Yang H, Feng Y, Sun X, et al: Enteral versus parenteral nutrition: effect on intestinal barrier function. Ann NY Acad Sci 2009;1165: 338-346.

5 Thompson JC: Burn and starvation increase programmed cell death in small bowel epithelial cells. Dig Dis Sci 2000;45:415-420.

6 Asfar S, Zhong R, Grant D: Small bowel transplantation. Surg Clin North Am 1994;74: 1197-1210.

7 Cui L, Takagi Y, Wasa M, et al: Nitric oxide synthase inhibitor attenuates intestinal damage induced by zinc deficiency in rats. J Nutr 1999;129:792-798.

8 Wang B, Wu G, Zhou Z, et al: Glutamine and intestinal barrier function. Amino Acids 2015;47:2143-2154.

9 Lightfoot E Jr, Horton JW, Maass DL, et al: Major burn trauma in rats promotes cardiac and gastrointestinal apoptosis. Shock 1999; 11:29-34.

10 Holt PR, Moss SF, Heydari AR, et al: Diet restriction increases apoptosis in the gut of aging rats. J Gerontol A Biol Sci Med Sci 1998; 53:B168-B172.
11 Quigley EMM: Leaky gut-concept or clinical entity? Curr Opin Gastroenterol 2016;32:7479.

12 Yamamoto S, Tanabe M, Wakabayashi G, et al: The role of tumor necrosis factor- $\alpha$ and interleukin- $1 \beta$ in ischemia-reperfusion injury of the rat small intestine. J Surg Res 2001;99: 134-141.

13 Booth C, Potten CS: Gut instincts: thoughts on intestinal epithelial stem cells. J Clin Invest 2000;105:1493-1499.

14 Song X, Dai D, He X, et al: Growth factor FGF2 cooperates with interleukin-17 to repair intestinal epithelial damage. Immunity 2015;43:488-501

15 van Landeghem L, Santoro MA, Mah AT, et al: IGF1 stimulates crypt expansion via differential activation of 2 intestinal stem cell populations. FASEB J 2015;29:2828-2842.

16 Howe KL, Lorentz RJ, Assa A: Transforming growth factor- $\beta 1$ protects against intestinal epithelial barrier dysfunction caused by hypoxia-reoxygenation. Shock 2015;43:483489.

17 Ko TC, Bresnahan WA, Thompson EA: Intestinal cell cycle regulation; in Meijer L, Guidet S, Philippe M (eds): Progress in Cell Cycle Research. New York, Plenum Press, 1997, pp 43-52.

18 Chowdhury A, Fukuda R, Fukumoto S: Growth factor mRNA expression in normal colorectal mucosa and in uninvolved mucosa from ulcerative colitis patients. J Gastroenterol 1996;31:353-360.
19 Beck PL, Podolsky DK: Growth factors in inflammatory bowel disease. Inflamm Bowel Dis 1999;5:44-60.

20 Boenisch T (ed): Handbook: Immunochemical Staining Methods, ed 3. Carpinteria, DAKO, 2001. www.ihcworld.com/_books/ Dako_Handbook.pdf.

21 Ito J, Uchida H, Yokote T, et al: Fasting-induced intestinal apoptosis is mediated by inducible nitric oxide synthase and interferon- $\gamma$ in rat. Am J Physiol Gastrointest Liver Physiol 2010;298:G916-G926.

22 Fu X, Sheng Z, Wang Y, et al: Basic fibroblast growth factor reduces the gut and liver morphologic and functional injuries after ischemia and reperfusion. J Trauma 1997;42: 1080-1085.

23 Cuevas P, Reimers D, Carceller F, et al: Fibroblast growth factor-1 prevents myocardial apoptosis triggered by ischemia reperfusion injury. Eur J Med Res 1997;2:465-468.

24 Shin ED, Estall JL, Izzo A, et al: Mucosal adaptation to enteral nutrients is dependent on the physiologic actions of glucagon-like peptide-2 in mice. Gastroenterology 2005;128: 1340-1353.

25 Dahly EM, Guo Z, Ney DM: Alterations in enterocyte proliferation and apoptosis accompany TPN-induced mucosal hypoplasia and IGF-1-induced hyperplasia in rats. J Nutr 2002;132:2010-2014.

26 Lin RL, Zhao LJ: Mechanistic basis and clinical relevance of the role of transforming growth factor- $\beta$ in cancer. Cancer Biol Med 2015; 12:385-393. 